\title{
Novel Magnified Single-Balloon Enteroscopy Enables Observation of Jejunal White Spots Associated with Lymphangiectasia
}

\author{
Kentaro Tominaga Atsunori Tsuchiya Yuzo Kawata Junji Yokoyama \\ Shuji Terai
}

Division of Gastroenterology and Hepatology, Graduate School of Medical and Dental Sciences, Niigata University, Niigata, Japan

\section{Keywords}

Jejunal lesion · Magnifying enteroscopy · Primary intestinal lymphangiectasia $\cdot$ Single-balloon enteroscopy $\cdot$ Villi

\begin{abstract}
A 59-year-old woman was diagnosed with primary intestinal lymphangiectasia (PIL), with characteristic findings on capsule enteroscopy and confirmation by histopathological examination of biopsy specimens. We viewed the abnormal jejunal mucosa using a newly developed magnifying singleballoon enteroscope (SIF-Y0007). Conventional observation showed leakage of chyle. However, using this new scope, we could see scattered white villi, representing dilated lymphatic vessels within the intestinal villi protruding from the dilated submucosal lymphoid vessels (D2-40 positive) within an edematous jejunal lesion. This report is the first to describe the white villi in a patient with PIL observed clearly using a newly developed magnifying enteroscope. Technological advancements and the accumulation of reported pathological data would further improve our understanding of the pathophysiological aspects of this disease entity, even in the jejunum.

(c) 2018 S. Karger AG, Basel
\end{abstract}

\section{Background}

Primary intestinal lymphangiectasia (PIL) is a rare disorder with an unknown underlying etiology, defined in 1961 by Waldmann et al. [1]. PIL, or Waldmann disease, is characterized by impaired intestinal lymphatic drainage due to primary ectasia (diffuse or localized to the intestinal lymphatic vessels). In contrast, secondary intestinal lymphangiectasia is due to secondary impacts on the normal lymph circulation, such as those in heart disease and retroperitoneal lymph node enlargements [2]. Although PIL mostly affects children, it is not exclusively a childhood disease, and the age of disease onset varies. The classical symptoms of PIL include intermittent diarrhea and lower limb edema, accompanied by other signs of malnutrition and hypoalbuminemia [3]. The histopathological findings of PIL are characterized by dilated and tortuous vessels in the small bowel mucosa and submucosa, resulting in protein-losing enteropathy and its sequela [4]. In this review, we describe the white villi within an edematous jejunal lesion in a patient with PIL, observed using a prototype magnifying single-balloon enteroscope (SIF Y-0007, Olympus). We believe the find-

\section{KARGER}

(c) 2018 S. Karger AG, Basel

E-Mail karger@karger.com

www.karger.com/ddi
Atsunori Tsuchiya

Division of Gastroenterology and Hepatology

Graduate School of Medical and Dental Sciences, Niigata University

1-757 Asahimachi-dori, Chuo-ku, Niigata 9518510 (Japan)

E-Mail atsunori@med.niigata-u.ac.jp 

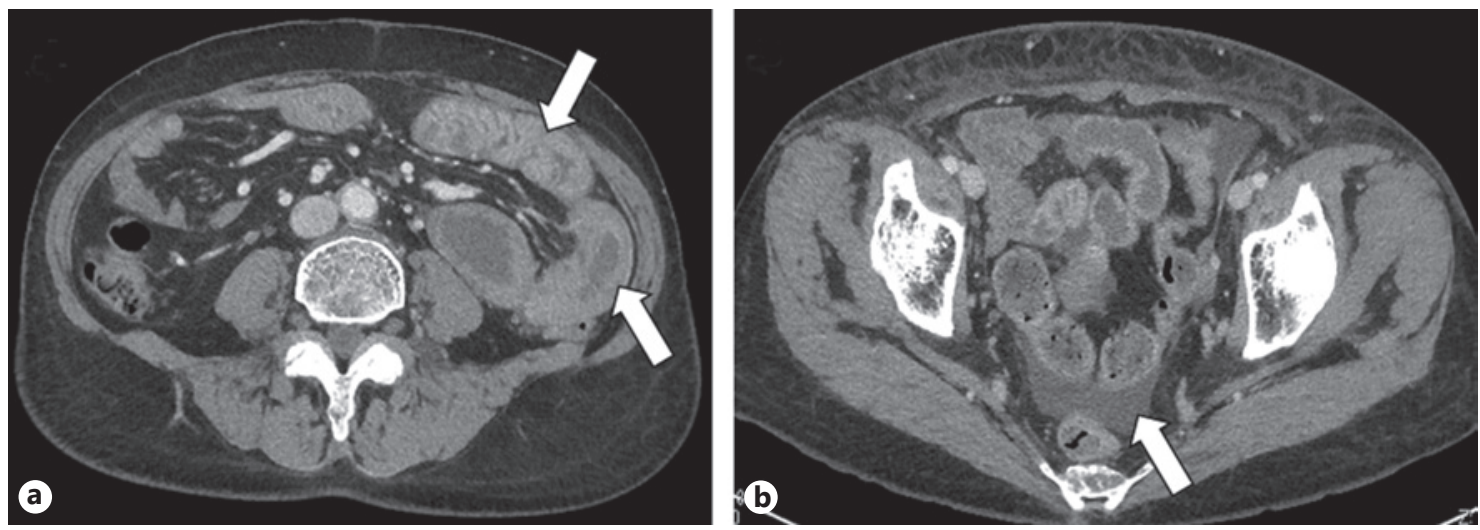

Fig. 1. a, b Computed tomography. Thickening of the wall of the small intestine is detected (white arrows).
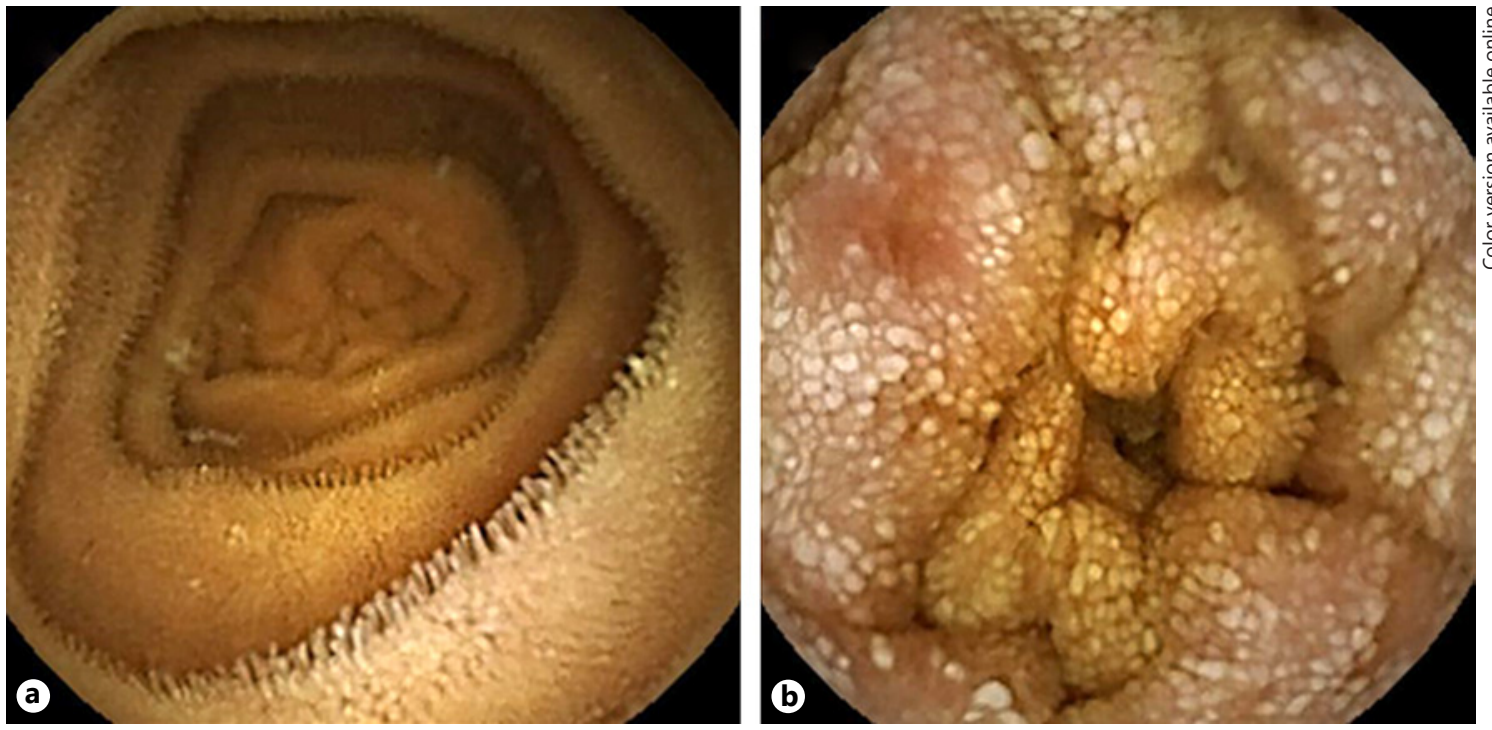

Fig. 2. Findings on capsule endoscopy. Almost normal findings are observed in the duodenum (a). Scattered white spots, with a characteristic snowflake appearance, are detected in the jejunum (b).

ings of the present study are of particular importance, as only a few previous reports have described the small intestinal lesions in PIL using magnifying enteroscopy.

\section{Case Report}

We present the case of 59-year-old woman admitted to our hospital with complaints of diarrhea and lower leg edema. She had intermittent episodes of occasional bilateral perimalleolar edema and diarrhea for 9 years, accompanied by slight abdominal pain and nausea. Malabsorption symptoms (anemia, hypocalcemia, and hypoproteinemia) gradually worsened over a few months prior to admission. She had no history of surgery and there was no significant family history. Her previous medical history was unremarkable.

Novel Magnified Single-Balloon Enteroscopy
On physical examination, only lower leg edema was present. Laboratory findings on admission were as follows: decreased hemoglobin $(11.0 \mathrm{~g} / \mathrm{dL})$, total protein $(48 \mathrm{~g} / \mathrm{L})$, albumin $(2.3 \mathrm{~g} / \mathrm{dL})$, globulin $(11.4 \mathrm{~g} / \mathrm{L})$, serum calcium $(8.1 \mathrm{mg} / \mathrm{dL})$, and immunoglobulin (IgG, $424 \mathrm{mg} / \mathrm{dL}$; IgM, $14 \mathrm{mg} / \mathrm{dL}$; and IgA, $63 \mathrm{mg} / \mathrm{dL}$ ) levels and high fecal anti-trypsin clearance $(43.3 \mathrm{~mL} /$ day). Tumor marker levels, including interleukin-2 receptor levels, were within the reference ranges. Additional tests were performed to exclude secondary intestinal lymphangiectasia. Immunological and thyroid disorders, tuberculosis, renal and hepatic impairment, and human immunodeficiency virus were also excluded.

Multi-slice CT of the chest and abdomen showed thickening of the small intestinal wall and ascites; along with the clinical findings, there were no signs of lymphoma, lymphadenopathy, or tumors (Fig. 1a, b). Abdominal doppler ultrasound showed no signs of portal hypertension or compromised mesenteric venous circulation. Upper endoscopy showed only mucosal changes character- 

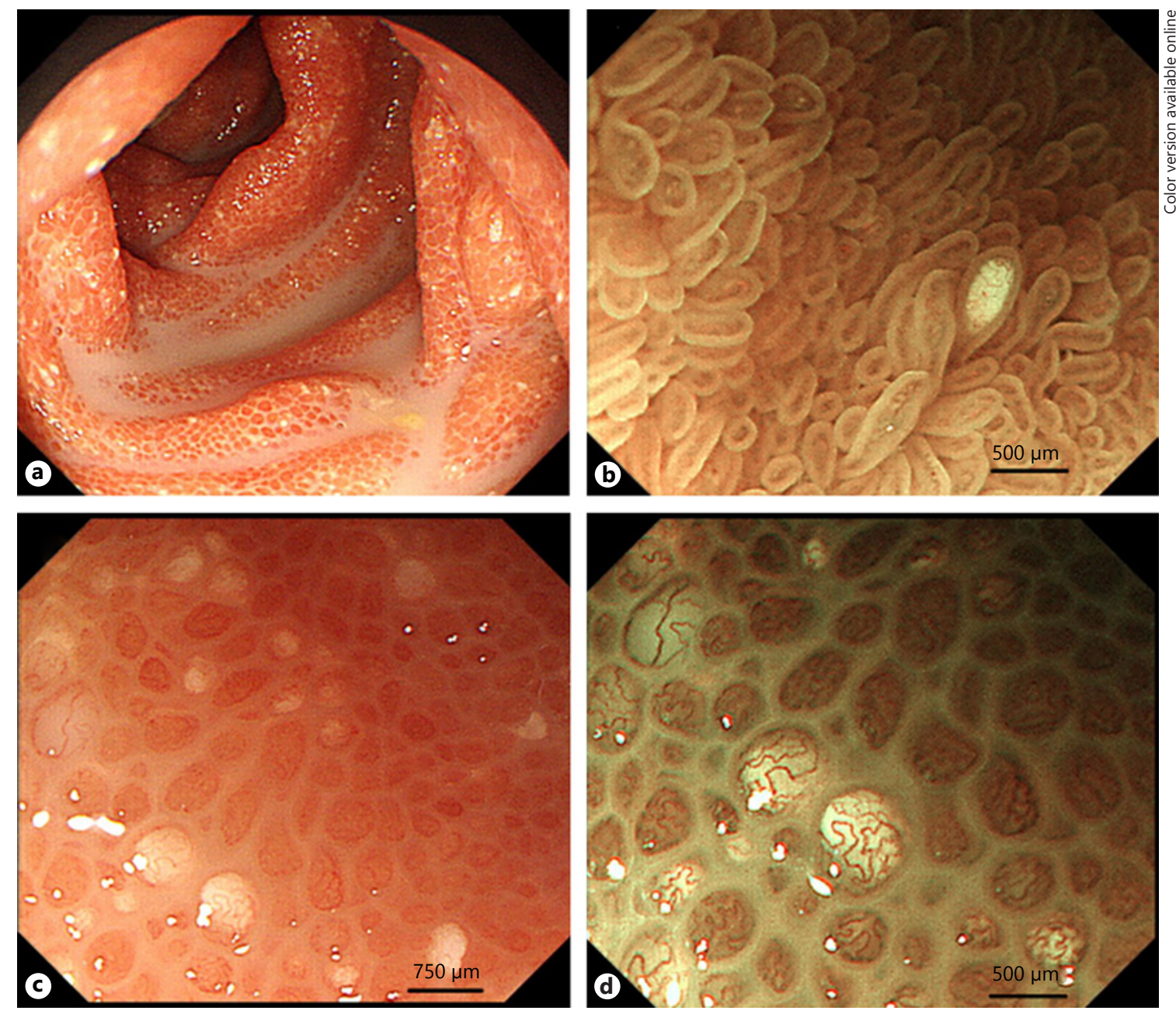

Fig. 3. Findings on single-balloon enteroscope (SIF-Y0007, Olympus). Conventional observation shows leakage of chyle in the jejunum (a). A magnified view shows single white intestinal villi in a normal-appearing duodenum (b). Scattered white villi overlying the edematous and hyperemic jejunal mucosa are observed (c). Scattered white spots within the tips of villi and the regular caliber of loop-like capillaries are observed (d).

pus). Conventional observation showed leakage of chyle (Fig. 3a), and a super-magnified view showed individual white intestinal villi in the normal-appearing duodenum (Fig. 3b). Scattered white irregular villi structure overlying the edematous jejunal mucosa were observed, highly suggestive of intestinal lymphangiectasia (Fig. 3c). Tortuous regular capillaries were seen on the surface of the swollen villi under magnified observation (Fig. 3d).

To the best of our knowledge, there are few previous reports describing small intestinal lesions in PIL using magnifying enteroscopy. Although in 2015 Iwamuro et al. [10] reported magnified endoscopic findings of intestinal lymphangiectasis, recent technical advances in endoscopy allow a clearer view.

Histological analysis revealed that the scattered white spots reflected the pathological dilatation of the lymphatic ducts, which were positive for D2-40. This analysis is consistent with that in a previous report [11].

In the present case, the histology of the intestinal biopsy specimens demonstrated dilated lymphatic vessels within the intesti- 


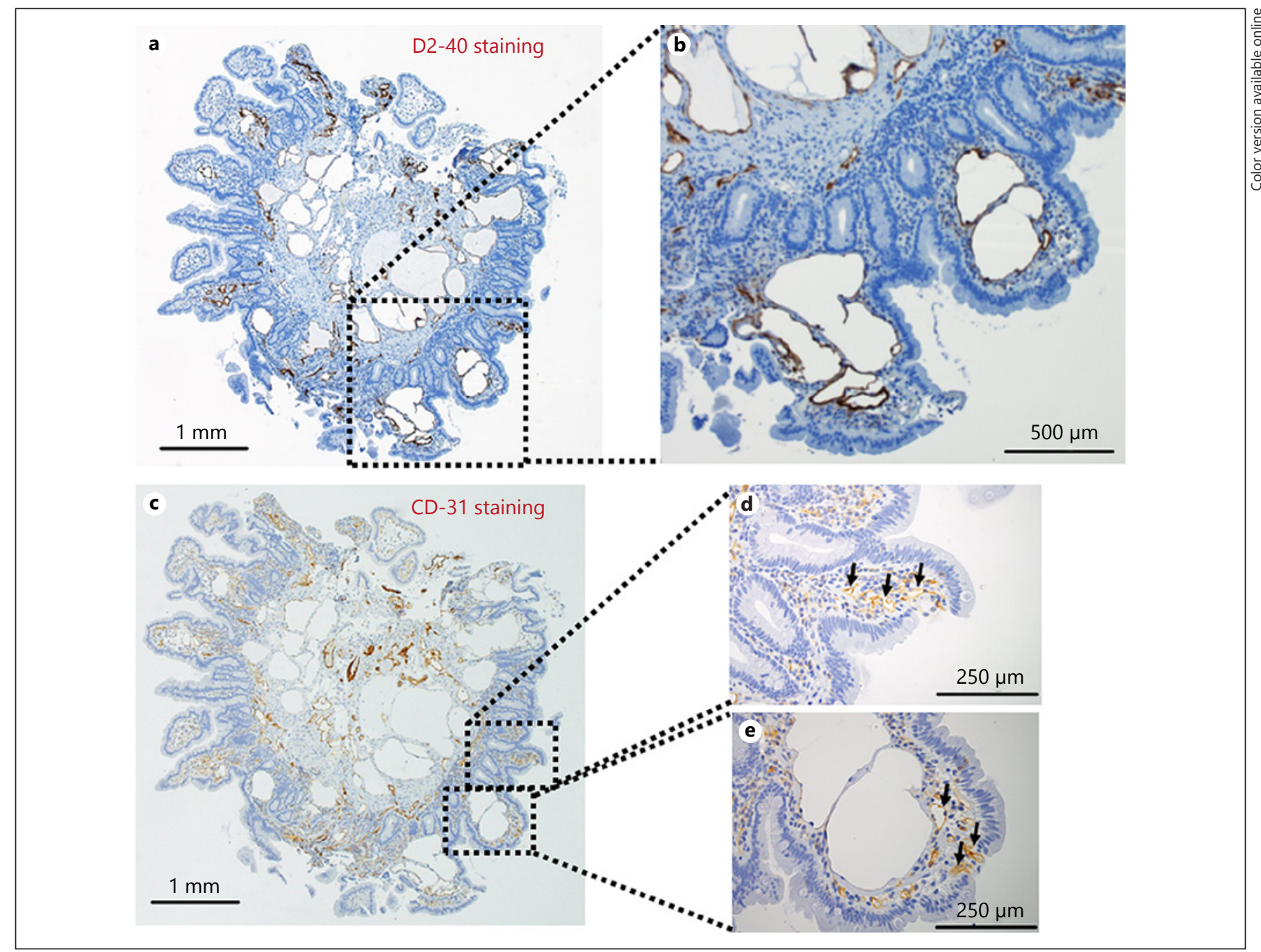

Fig. 4. Histopathological analysis. D2-40 and CD-31 immunohistochemistry of the intestinal biopsy specimens is shown. Dilated lymphatics in the mucosa and submucosa is observed (a: original magnification $\times 100)$. Dilated mucosal lymphoid vessels (D2-40-positive) of the intestinal villi within the edematous jejunal lesion are shown (b: original magnification $\times 400$ ). CD-31-positive vessel in the mucosa and submucosa are observed (c: original magnification $\times 100)$. Scattered or compressed mucosal vessels (CD31-positive) within normal villi or swollen villi are shown (d, e: original magnification $\times 400$ ). Black arrows show CD-31-positive vessels. nal villi, protruding from the dilated submucosal lymphoid vessels, which were positive for lymphatic endothelial marker D240 within an edematous jejunal lesion (Fig. 4a, b). The slice was also stained for vascular endothelial cell marker CD-31 (Fig. 4c). In the normal villi, CD-31-positive endothelial cells were scattered (Fig. 4d). On the other hand, in the whitish swollen villi, CD-31-positive endothelial cells were compressed to the surface by the dilated lymphoid duct (Fig. 4e). Therefore, regular capillaries could be seen clear of the swollen villi by magnified endoscopy.

Although several therapeutic management strategies for PIL have been proposed, no standardized algorithms exist. The principles of the treatment of PIL are different from those of other forms of protein-losing enteropathy, with no exact recommendations on the dosage and duration of therapy. A high-protein, low- fat diet, supplemented with medium-chain triglycerides, is currently the most effective and widely prescribed treatment, with minimal side effects [12]. In the present case, a low-fat diet, supplemented with medium-chain triglycerides, vitamins, and electrolyte supplements was introduced, with subsequent improvements in her clinical (abdominal pain and diarrhea, cured; lower leg edema, relieved) and laboratory data (hemoglobin $[14.7 \mathrm{~g} / \mathrm{dL}$, total protein $[5.1 \mathrm{~g} / \mathrm{L}]$, albumin $[2.6 \mathrm{~g} / \mathrm{dL}]$, serum calcium $[8.7 \mathrm{mg} /$ $\mathrm{dL}]$ ).

In the present case, we performed a detailed observation and obtained biopsies using the SIF-Y0007. The magnified observation of the small intestine was first reported in 1980 by Tada et al. [13]; however, the scope (SIF-M, Olympus) utilized required insertion via the ropeway method and was therefore not widely used. In contrast, the SIF-Y0007 provides up to 80 times magnification and has 
an outer diameter of $9.9 \mathrm{~mm}$, with approximately the same contour as the typically used SIF-Q260, which has an outer diameter of 9.2 $\mathrm{mm}$. We did not experience greater difficulties in regard to the insertion technique and operability compared to those for a conventional examination of the small intestine.

Magnified observation can demonstrate a detailed morphology of the villus or the loop vessels. The relationship between magnified endoscopic findings and histological findings in PIL has not been previously reported. Scattered white spots and regular capillaries that were compressed to the surface by the dilated lymphoid duct were clearly observed by magnified endoscopy. Additionally, there have been no reports of small bowel lesions viewed using the SIF-Y0007 in cases of lymphangiectasia. Further analysis on the usefulness of magnified endoscopy as a diagnostic tool for intestinal pathogenesis is necessary. However, a detailed observation using the SIF-Y0007, combined with pathological findings, would increase our understanding of PIL.

\section{Disclosure Statement}

The authors declare that they have no conflicts of interest to disclose.

\section{Informed Consent Statement}

Written informed consent was obtained from the patient for publication of this case report and accompanying images.

\section{Authors Contribution}

K.T., A.T., Y.K., and J.Y. made the diagnosis and drafted the manuscript. S.T. analyzed data. All 5 authors critically reviewed the manuscript and approved of the final draft.

\section{References}

1 Waldmann TA, Steinfeld JL, Dutcher TF, Davidson JD, Gordon RS Jr: The role of the gastrointestinal system in "idiopathic hypoproteinemia”. Gastroenterology 1961;41:197207.

2 Vignes S, Carcelain G: Increased surface receptor fas (CD95) levels on CD4+ lymphocytes in patients with primary intestinal lymphangiectasia. Scand J Gastroenterol 2009;44: 252-256.

3 Vignes S, Bellanger J: Primary intestinal lymphangiectasia (Waldmann's disease). Orphanet J Rare Dis 2008;3:5.

4 Umar SB, DiBaise JK: Protein-losing enteropathy: case illustrations and clinical review. Am J Gastroenterol 2010;105:43-49.

5 Chamouard P, Nehme-Schuster H, Simler JM, Finck G, Baumann R, Pasquali JL: Videocapsule endoscopy is useful for the diagnosis of intestinal lymphangiectasia. Dig Liver Dis 2006;38:699-703.

6 Lai Y, Yu T, Qiao XY, Zhao LN, Chen QK: Primary intestinal lymphangiectasia diagnosed by double-balloon enteroscopy and treated by medium-chain triglycerides: a case report. J Med Case Rep 2013;7:19.

7 Oh TG, Chung JW, Kim HM, Han SJ, Lee JS, Park JY, Song SY: Primary intestinal lymphangiectasia diagnosed by capsule endoscopy and double balloon enteroscopy. World J Gastrointest Endosc 2011;3:235-240.

8 Alshikho MJ, Talas JM, Noureldine SI, et al: Intestinal lymphangiectasia: insights on management and literature review. Am J Case Rep 2016; 17:512-522.

9 Siddeswari R, Manohar S, Reddy T, et al: Primary intestinal lymphangiectasia. J Indian Acad Clin Med 2016;17:60-63.
10 Iwamuro M, Okada H, Takata K, et al: Magnified endoscopic features of duodenal follicular lymphoma and other whitish lesions. Acta Med Okayama 2015;69:37-44.

11 Asakura H, Miura S, Morishita T, Aiso S, Tanaka T, Kitahora T, Tsuchiya M, Enomoto Y, Watanabe Y: Endoscopic and histopathological study on primary and secondary intestinal lymphangiectasia. Dig Dis Sci 1981;26: 312-320.

12 Desai AP, Guvenc BH, Carachi R: Evidence for medium chain triglycerides in the treatment of primary intestinal lymphangiectasia. Eur J Pediatr Surg 2009; 19:241-245.

13 Tada M, Suyama Y, Shimizu T, Fujii H, Miyoshi M, Nishimura S, Nishitani T, Katake K, Shimono M, Askasaka Y, Kawai K: Observation of villi with the magnifying entero-colonoscopes. Gastroenterol Endosc 1980;22:647-654. 